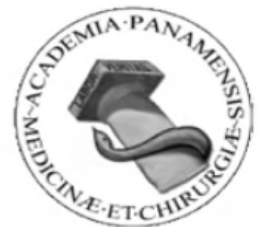

Artículo original

\title{
Experiencia En El Manejo De Pacientes Con Cáncer De Pulmón Avanzado Alk+ En El Instituto Oncológico Nacional De Panamá.
}

\section{Experience in the Management of Patients with Advanced Lung Cancer Alk + at the National Oncology Institute of Panama.}

\author{
*Sowley Taysser, ${ }^{* *}$ Moreno-Ríos Joel, ${ }^{* * *}$ Vergara Ruth, ${ }^{* *}$ Araúz Erik \\ *Médico Residente de Oncología Médica, ${ }^{* *}$ Médico especialista, Oncología Médica, ${ }^{* * *}$ Médico especialista, Patología Quirúrgica, \\ Instituto Oncológico Nacional, Panamá.
}

\section{Palabras claves: rearreglo, cáncer de pulmón, inhibidores de ALK.}

\section{Keywords:} rearrangement, lung cancer, ALK inhibitors.

Correspondencia a: Dr. Taysser Sowley Correo electrónico: taysser 26 @hotmail.com

Los autores declaran no tener conflicto de intereses en la publicación del presente trabajo.

\section{Resumen}

Introducción: el rearreglo de ALK está presente en 3\%-7\% de los pacientes con cáncer de pulmón avanzado. Los inhibidores de ALK constituyen el tratamiento de primera línea. El crizotinib fue el primer inhibidor aprobado. Metodología: Realizamos una revisión retrospectiva de los expedientes médicos de los pacientes con cáncer de pulmón ALK positivo desde febrero de 2014 hasta mayo de 2018. El objetivo era describir la incidencia, características clínico patológicas y determinar la supervivencia libre de progresión con el uso de crizotinib en primera línea. Resultados: De 406 pacientes evaluados, 26 resultaron positivos para ALK, obteniendo una incidencia de $6,4 \%$. La edad media de diagnóstico fue 64 años $(37-85)$ y 53,1\% fueron mujeres. El 92,4\% de los pacientes tenían ECOG 0 - 2. Un $43,2 \%$ con antecedente de tabaquismo. Todos los pacientes fueron diagnosticados con enfermedad al menos localmente avanzada y un $73,1 \%$ tenían enfermedad etapa IV. Al diagnóstico, 2 pacientes debutaron con metástasis cerebrales $(10,3 \%)$ y ambos recibieron RTHC. Adenocarcinoma fue la histología predominante $(96.3 \%)$. Un $86,9 \%$ de los pacientes iniciaron crizotinib de primera línea. La supervivencia libre de progresión fue 10.9 meses (IC 95\% 7,69-14,12). Los efectos adversos más comunes fueron las náuseas, diarrea, disgeusia y transaminitis, en su mayoría G1. Sin toxicidad G3 o superior. La supervivencia global de los pacientes expuestos a crizotinib fue de 16.9 meses (IC 95\% $11.29-21.50$ ).

Conclusión: Las características de los pacientes ALK positivo, así como la PFS asociada al crizotinib de primera línea en nuestra institución, es similar a la reportada en los estudios internacionales de fase III.

\section{Abstract}

Introduction: the rearrangement of ALK is present in 3\%-7\% of patients with advanced lung cancer. ALK inhibitors constitute the first line treatment. Crizotinib was the first approved inhibitor. Methodology: We conducted a retrospective review of medical records of patients with ALK positive lung cancer from February 2014 to May 2018. The objective was to describe the incidence, clinical pathological characteristics and determine progression-free survival with the use of crizotinib First line. Results: Of 406 patients evaluated, 26 were positive for ALK, obtaining an incidence of $6.4 \%$. The mean age of diagnosis was 64 years $(37-85)$ and $53.1 \%$ were women. $92.4 \%$ of patients had ECOG $0-2$. $43.2 \%$ had a history of smoking. All patients were diagnosed with at least locally advanced disease and $73.1 \%$ had stage IV disease. At diagnosis, 2 patients started with brain metastases $(10.3 \%)$ and both received RTHC. Adenocarcinoma was the predominant histology (96.3\%). 86.9\% of patients started crizotinib first line. The progression-free survival was 10.9 months $(95 \% \mathrm{Cl} 7.69$ 14.22). The most common adverse effects were nausea, diarrhea, dysgeusia and transaminitis, mostly G1. No toxicity G3 or higher. The overall survival of patients exposed to crizotinib was 16.9 months (95\% Cl $11.29-21.50)$.

Conclusion: The characteristics of the positive ALK patients, as well as the PFS associated with the first line crizotinib in our institution, is similar to that reported in the international phase III studies. 


\section{INTRODUCCIÓN}

servicio de patología de los pacientes diagnosticados con cáncer de pulmón ALK-positivo.

Como objetivo primario se estableció determinar la supervivencia libre de progresión (PFS) de los pacientes expuestos a Crizotinib en primera línea y como objetivos secundarios: la supervivencia global (OS), tasa de respuesta y control de la enfermedad con crizotinib, además del perfil de toxicidades. La valoración de la respuesta se realizó según el criterio de RECIST, reportado con las tomografías de evaluación. Los efectos adversos fueron clasificados y graduados según los criterios de terminología común para efectos adversos, versión 4.0 del NIH. El crizotinib fue utilizado a dosis de $250 \mathrm{mg}$ vía oral, dos veces al día. do a debutar en etapas avanzadas [5]. A principios de siglo $\mathrm{XXI}$, la quimioterapia representaba la única opción de tratamiento sistémico paliativo con supervivencia global media de aproximadamente 8 meses, mediana para progresión entre 3 a 4 meses y tasas de respuesta de $20 \%$ [6]. Sin embargo, se logran descubrir mutaciones conductoras/activadoras, entre ellas: el rearreglo de la proteína de fusión Anaplastic Lymphoma Kinasa (ALK), convirtiendo su inhibición en blanco terapéutico.

En el 2007, se hace la primera asociación con cáncer de pulmón en Japón, particularmente el reordenamiento ALK-EML4 $[7,8]$. Su incidencia es de aproximadamente $3 \%$ a $7 \%$ de todos los casos de cáncer de pulmón y entre sus características destacan: histología predominante adenocarcinoma, no fumadores o fumadores ligeros y pacientes jóvenes. Entre los métodos empleados para determinación del estado ALK actualmente, sobresalen las técnicas de hibridación in situ florescente (FISH) considerado el método de referencia y la inmunohistoquímica, con un valor predictivo positivo elevado.

El primer inhibidor de tirosina cinasa para ALK, crizotinib, logra su aprobación como estándar de primera línea, con la publicación del estudio de fase III 'PROFILE 1014' que demuestra la superioridad del crizotinib sobre la dupleta platino-pemetrexed con supervivencia libre de progresión (PFS) de 10.9 meses y tasa de respuesta (ORR) de $74 \%$ [13].

Posteriormente se han desarrollado otros inhibidores de ALK, como el ceritinib [14] y el alectinib [15, 16]. En Panamá contamos con el crizotinib como agente de primera línea. El objetivo de este estudio es describir nuestra experiencia en la incidencia, características clinicopatológicas y supervivencia libre de progresión con el uso de crizotinib en primera línea, en nuestros pacientes ALKpositivo.

\section{MATERIALES Y MÉTODOS}

Realizamos un estudio de tipo longitudinal retrospectivo, entre el período de febrero de 2014 a mayo de 2018, creando la base de datos con la información obtenida de los expedientes clínicos electrónicos y los registros del
Definimos supervivencia libre de progresión (PFS) como el tiempo entre el inicio del crizotinib hasta la progresión (clínica o radiográfica) o muerte. Supervivencia global (OS) como el tiempo del inicio del crizotinib hasta la muerte por cualquier causa.

\section{Determinación del estado ALK}

Desde el 2014 hasta mediados del 2016, la determinación se realizaba por medio de la prueba de hibridación in situ fluorescente (FISH) sobre el tejido fijado con formalina y embebido en parafina, con la sonda Vysis ALK break apart de la compañía Abbott. Desde mediados del 2016 a la actualidad, se utiliza la determinación por medio de Inmunohistoquímica con anticuerpos que se unen a la porción distal del dominio tirosina cinasa de ALK, conservado en todos los reordenamientos. Se utiliza el anticuerpo de la clona D5F3 para el equipo Ventana.

\section{Análisis Estadístico}

La elaboración de la base de datos y el análisis estadístico se realizaron utilizando el programa SPSS versión 22.0. Las características clinicopatológicas de los pacientes y el perfil de toxicidad se reportaron utilizando frecuencias. Para el cálculo de la PFS y OS se utilizó el método de Kaplan-Meier. La prueba de log Rank se utilizó para evaluación de variables asociadas a PFS.

\section{RESULTADOS}

Entre febrero de 2014 y mayo de 2018, se evaluaron 406 pacientes con cáncer de pulmón y 26 resultaron ALK positivo, obteniendo una incidencia de $6.4 \%$. Un $50 \%$ de las determinaciones se realizó con FISH y el resto con IHQ. La edad media fue de 65 años y un $42.3 \%$ tenían antecedente de tabaquismo. Las características generales de los pacientes se resumen en la tabla 1.

Dentro del $73.1 \%$ (19/26) pacientes que debutaron con enfermedad metastásica, 7 pacientes $(29,2 \%)$ se presentaron con enfermedad a múltiples sitios. El pulmón contralateral $(66,7 \%)$, el hueso $(29,2 \%)$ y el hígado $(16.7 \%)$ fueron los sitios más frecuentemente afectados. Un 
Sowley y cols., Cáncer de Pulmón ALK positivo en el ION.

Tabla 1. Características de los Pacientes

\begin{tabular}{lc}
\multicolumn{1}{c}{ Características } & Frequencia (N = 26) \\
\hline Edad & 64 \\
$\quad$ Media & $37-85$ \\
Rango & $12(46,2 \%)$ \\
Sexo - No. (\%) & $14(53,8 \%)$ \\
$\quad$ Masculino & \\
Femenino & $20(77,0 \%)$ \\
ECOG - No. (\%) & $4(15,4 \%)$ \\
$0-1$ & $2(7,7 \%)$ \\
2 & $11(42,3 \%)$ \\
3 & $25(96,2 \%)$ \\
Tabaquismo - No. (\%) & $1(3,8 \%)$ \\
Histología - No. (\%) & $7(26,9 \%)$ \\
Adenocarcinoma & $19(73,1 \%)$ \\
Pobremente Diferenciado & \\
Etapa - No. (\%) & $20(86,9 \%)$ \\
Localmente Avanzado & $3(13,1 \%)$ \\
Metastásico & $2 / 19(10,5 \%)$ \\
Tratamiento de 1ra. Línea & $2 / 2(100 \%)$ \\
- No. (\%) & \\
Crizotinib & QT
\end{tabular}

Gráfica 1. Supervivencia libre de progresión (PFS) con crizotinib en primera línea.

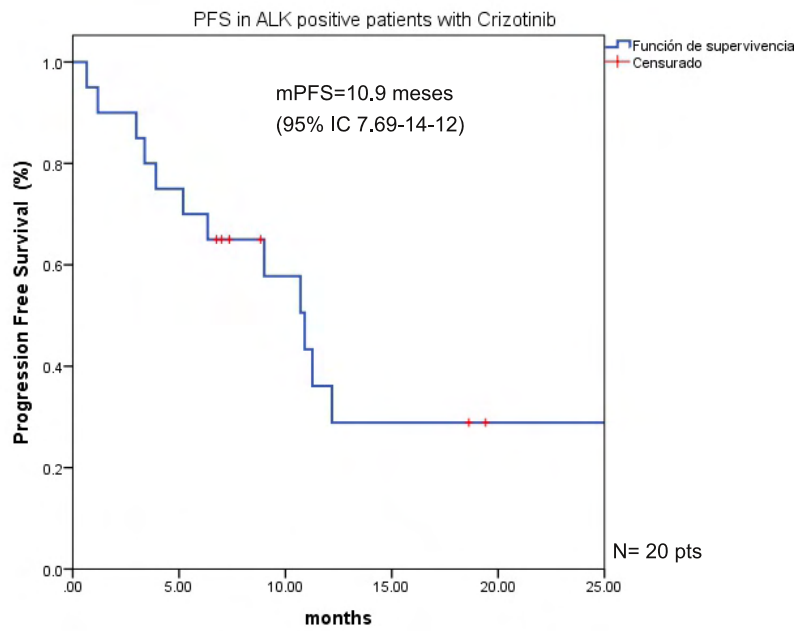

$10.5 \%(2 / 19)$ se presentaron con enfermedad metastásica cerebral al diagnóstico, recibiendo tratamiento con radioterapia holocraneana.

Un $88,5 \%$ lograron iniciar algún tipo de tratamiento sistémico de primera línea y específicamente $86,9 \%(20 / 26)$ iniciaron con crizotinib. La mediana de PFS fue de 10.9 meses (IC 95\% 7,69 - 14,12). (Ver Gráfica 1).

Se evaluaron posibles factores asociados a la PFS, pero no se encontró asociación estadísticamente significativa (Ver Tabla 2). Del 75\% (15/20) de pacientes que progresaron, $40 \%(6 / 15)$ pudieron iniciar una segunda línea con quimioterapia estándar y un 20\% (3/15) logra- Tabla 4).
Tabla 2. Factores Asociados a la PFS del Crizotinib de Primera Línea

\begin{tabular}{lll}
\multicolumn{1}{c}{ Factor } & HR & P \\
\hline $\begin{array}{l}\text { Sexo } \\
\text { Femenino vs } \\
\text { Masculino }\end{array}$ & 2.018 & \\
$\begin{array}{l}\text { ECOG } \\
\quad \text { o=2 vs 0-1 }\end{array}$ & 3.479 & 0.155 \\
$\begin{array}{l}\text { Tabaquismo } \\
\text { No vs Sí }\end{array}$ & 0.362 & 0.062 \\
$\begin{array}{l}\text { Mets. Al SNC } \\
\text { No vs Sí }\end{array}$ & 0.071 & 0.547 \\
$\begin{array}{l}\text { Presentación } \\
\text { Etapa II-III vs IV }\end{array}$ & 0.016 & 0.790 \\
$\begin{array}{l}\text { Edad } \\
<65 a \text { vs >65a }\end{array}$ & 0.395 & 0.901 \\
\hline
\end{tabular}

Tabla 3. Evaluación de la respuesta al Crizotinib

\begin{tabular}{|c|c|c|}
\hline \multicolumn{2}{|r|}{ Respuesta } & Frecuencia \\
\hline \multirow{4}{*}{ 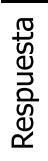 } & Completa & $1(5 \%)$ \\
\hline & Parcial & $5(25 \%)$ \\
\hline & Estable & $9(45 \%)$ \\
\hline & Progresión & $5(25 \%)$ \\
\hline \multicolumn{2}{|c|}{ Tasa de Respuesta Objetiva } & $6(30 \%)$ \\
\hline \multicolumn{2}{|c|}{$\begin{array}{l}\text { Control de la } \\
\text { Enfermedad/Beneficio Clínico }\end{array}$} & $15(75 \%)$ \\
\hline
\end{tabular}

Gráfica 2. Superviviencia global de los pacientes con crizotinib en primera línea.



ron recibir ceritinib en tercera línea. La mediana de supervivencia global media para los pacientes que iniciaron tratamientos sistémicos de primera línea fue de 21.8 meses (IC 95\% 13.02 - 30.56) y específicamente con crizotinib fue de 16.9 meses (IC 95\% 11.29 - 21.50). (Ver Gráfica 2).

Se obtuvo una tasa de respuesta objetiva de $30 \%$ y un beneficio clínico de $75 \%$ con el uso de crizotinib (Ver Tabla 3).

Entre los efectos adversos más reportados se describen las náuseas, la diarrea, la disgeusia y las transminitis, en su mayoría G1. No hubo toxicidades G3 o mayores (Ver 
Tabla 4. Perfil de efectos adversos asociados al Crizotinib

\section{Toxicidad}

Frecuencia Crizotinib ( $\mathbf{N}=20 p x)$ G1 o G2

\begin{tabular}{lc}
\hline Náuseas & $7(35 \%)$ \\
Diarrea & $6(30 \%)$ \\
Vómitos & $1(5 \%)$ \\
Disgeusia & $3(15 \%)$ \\
Transaminitis & $2(10 \%)$ \\
Astenia & $1(5 \%)$ \\
Fotosensibilidad & $1(5 \%)$ \\
Edema & $1(5 \%)$ \\
Dispepsia & $1(5 \%)$ \\
\hline
\end{tabular}

\section{DISCUSIÓN}

El descubrimiento de las mutaciones conductoras marcó un hito en el manejo de los pacientes con cáncer de pulmón. En el grupo de pacientes con rearreglo de ALK, este trabajo representa el primer estudio realizado en nuestro país y uno de los primeros en la región, describiendo las características clinicopatológicas y parámetros de eficacia asociados al tratamiento específico. Nuestra incidencia de cáncer de pulmón ALK positivo de $6.4 \%$, coincide con lo reportado en los estudios de referencia, en un $3 \%$ a $7 \%$ de los casos $[10,19]$.

El estudio de Martin et al., es el primer estudio encontrado en nuestra revisión que se enfoca también en población latinoamericana y reporta igual incidencia que la nuestra [20]. Nuestras características clínicas son similares a lo reportado, con excepción de la edad media de 65 años, ligeramente mayor. Dado que un 42,3\% tuvieron antecedente de tabaquismo, se justifica la realización de la prueba también en fumadores y exfumadores.

El primer TKI aprobado como tratamiento de primera línea fue el crizotinib y es el agente con el que actualmente contamos en nuestra institución. Obtuvimos una PFS de 10.9 meses, consistente con lo demostrado en su estudio de aprobación [13], y similar también a estudios de poblaciones específicas (Asia, Italia), e incluso en el más reciente estudio (ALEX) con 11.1 meses, superado en comparación directa por el Alectinib [13, 16, 21, 22]. Llama la atención que en el estudio de Martin la PFS, se describe en 7.7 meses [20].

Una posibilidad para esta discrepancia, dado que compartimos características clínicas similares, es el acceso limitado a terapias específicas en algunas regiones de Latinoamérica [23]. En nuestra serie, más del $85 \%$ de los pacientes pudieron iniciar crizotinib en primera línea.

La tasa de respuesta objetiva fue de $30 \%$, inferior a los esperado [13], siendo el mayor control con enfermedad estable. No ser un ensayo prospectivo, con tiempos es- pecíficos de evaluación de respuesta y con un comité revisor, pueden influenciar este parámetro.

Nuestra OS de 16.9 meses no es como la actualmente reportada $[13,16]$. Entre posibles factores está el seguimiento riguroso que se lleva en los estudios prospectivos, las condiciones socioeconómicas de nuestros pacientes y principalmente, la exposición a otros inhibidores de ALK en líneas posteriores. Hasta el momento contamos con aprobación solo en primera línea. A pesar de esto, ya doblamos la OS tradicionalmente ofrecida por la quimioterapia [6].

El perfil de toxicidades fue similar a lo reportado en estudios internacionales previos $[12,13]$, todas de bajo grado. No hubo toxicidad G3 ni se requirió discontinuar el tratamiento por efectos adversos.

Como limitación de nuestro trabajo señalamos el hecho de ser un estudio retrospectivo, de un solo centro y con pocos pacientes (esperado por la incidencia). Sin embargo, constituye la primera revisión realizada en nuestra institución donde evaluamos eficacia de tratamientos innovadores en los pacientes ALK positivos y lo que ocurre en la práctica clínica usual.

Este trabajo es generador de hipótesis así como base para futuros estudios prospectivos, justificando la adquisición de nuevos inhibidores. Es también una de las primeras revisiones realizada como país en la región de Latinoamérica.

\section{CONCLUSIÓN}

Se observó que la incidencia, características clinicopatológicas y PFS con crizotinib de primera en línea en los pacientes con cáncer de pulmón ALK positivo del Instituto Oncológico Nacional de Panamá coincide con la reportada en la literatura internacional y los estudios pivótales.

\section{REFERENCIAS}

[1] Organización Mundial de la Salud (2012) - Cáncer [Internet]. Disponible en: http://www.who.int/cancer/about/facts/es/

[2] Siegel RL, Miller KD, Jemal A. Cancer Statistics, 2018. CA Cancer J Clin. 2018 Jan;68(1):7-30. Doi: 10.3322/caac.21442. Epub 2018 Jan 4.

[3] Organización Mundial de la Salud (2012) - GLOBOCAN - Cáncer de Pulmón en Las Américas [Internet]. Disponible en:

https://www.paho.org/hq/dmdocuments/2014/OPSNota-Informativa-Epi-Cancer-Pulmon-2014.pdf

[4] Registro Nacional del Cáncer de Panamá. Boletín 
Sowley y cols., Cáncer de Pulmón ALK positivo en el ION.

Estadístico 2014 - 2016 [Internet]. Tomado de: http://www.minsa.gob.pa/sites/default/files/general/boletin_2014_rncp_.pdf

[5] Tan WW. Non- Small Cell Lung Cancer [Internet]. eMedicine, Medscape. Jul 19, 2018. Disponible en: https://emedicine.medscape.com/article/279960overview

[6] Schiller JH, Harrington D, Belani CP et al. Comparison of four chemotherapy regimens for advanced non-small cell lung cancer. N Engl J Med. 2002 Jan 10;346(2):92-8.

[7] Shaw AT, Engelman JA. ALK in lung cáncer: past, present, and future. J Clin Oncol. 2013 Mar 10; 31(8):1105-11.

[8] Soda M, Choi YL, Enomoto $M$ et al. Identification of the transforming EML4-ALK fusion gene in nonsmall-cell lung cancer. Nature. 2013 Mar 10; 31(8):1105-11.

[9] Kim H, Chung JH. Overview of clinic pathologic features of ALK-rearranged lung adenocarcinoma and current diagnostic testing for ALK rearrangement. Transl Lung Cancer Res. 2015 Apr; 4(2): 149-155.

[10] Kwal EL, Bang YJ, Camidge DR, et al. Anaplastic lymphoma kinase inhibition in non-small-cell lung cancer. N Engl J Med. 2010 Oct 28; 363(18):1693-703.

[11] Blackhall F, Camidge DR, Shaw AT et al. Final results of the large-scale multinational trial PROFILE 1005: efficacy and safety of crizotinib in previously treated patients with advanced/metastatic ALK-positive non-small-cell lung cancer. ESMO Open. 2017 Aug 17; 2(3): e000219.

[12] Shaw AT, Kim DW, Nakagawa K, et al. Crizotinib vs chemotherapy in advanced ALK-positive lung cancer. N Engl J Med. 2013 Jun 20; 368(25):2385-94.

[13] Solomon BJ, Mok T, Kim DW, et al. First-Line crizotinib versus chemotherapy in ALK-positive lung cancer. N Engl J Med. 2014 Dec 4; 371(23):2167-77.

[14] Soria JC, Tan DSW, Chiari R, et al. First-line ceritinib versus platinum-based chemotherapy in advanced ALK-rearranged non-small-cell lung cancer (ASCEND-4): a randomized, open-label, phase 3 study. Lancet. 2017 Mar 4; 389(10072):917-929.

[15] Hida T, Nokihara H, Kondo M. Alectinib versus crizotinib in patients with ALK-positive non-small-cell lung cancer (J-ALEX): an open-label, randomised phase 3 trial. Lancet. 2017 Jul 1; 390(10089):29-39.
[16] Peters S, Camidge R, Shaw AT, et al. Alectinib versus Crizotinib in Untreated ALK-Positive Non-SmallCell Lung Cancer. N Engl J Med. 2017 Aug 31; 377(9):829-838.

[17] Conklin CM, Craddock KJ, Have C, et al. Immunohistochemistry is a Reliable Screening Tool for Identification of ALK Rearrangement in Non-SmallCell Lung Carcinoma and is Antibody Dependent. JThorac Oncol. 2013; 8: 45-51.

[18] Lindeman NI, Cagle PT, Beasley MB, et al. Molecular testing guideline for selection of lung cancer patients for EGFR and ALK tyrosine kinase inhibitors: guideline from the College of American Pathologists, International Association for the Study of Lung Cancer, and Association for Molecular Pathology. J Thorac Oncol 2013; 8: 823-859.

[19] Hofman P. ALK in Non-Small Cell Lung Cancer (NSCLC) Pathobiology, Epidemiology, Detection from Tumor Tissue and Algorithm Diagnosis in a Daily Practice. Cancers (Basel), 2017 Aug; 9 (8): 107.

[20] Martin C, Cardona AF, Zatarain-Barrón ZL, et al. Real-World Treatment Patterns, Survival, and Prediction of CNS Progression in ALK-Positive NonSmall-Cell Lung Cancer Patients treated with first-line Crizotinib in Latin America Oncology Practices. Oncology. 2018; 94(5):297-305.

[21] Lu S, Mok T, Lu Y, et al. Phase 3 study of first-line crizotinib vs pemetrexed- cisplatin/carboplatin (PCC) in East Asian patients with ALK+ advanced nonsquamous non-small cell lung cancer (NSCLC). J Clin Oncol 2016; 34: Suppl: 9058.

[22] De Carlo E, Del Savio MC, Polesel J, et al. Outcomes of ALK positive lung cancer patients treated with crizotinib or second-generation ALK inhibitor: a monoinstitutional experience. Oncotarget. 2018 Mar 16; 9(20): 15340-15349.

[23] Homedes N, Ugalde A. Availability and affordability of new medicines in Latin American countries where pivotal clinical trials were conducted. Bull World Health Organ. 2016 May 01; 94(5): 404. 